\title{
Detection of THz nanosecond pulses by a fast hot electron bolometer
}

\author{
Norbert Palka, ${ }^{* 1}$ Przemyslaw Zagrajek, ${ }^{1}$ Adam Czerwinski, ${ }^{2}$ Tomasz Trzcinski, ${ }^{1}$ Elzbieta Rurka ${ }^{1}$, \\ Mieczyslaw Szustakowski, ${ }^{1}$ and Maciej Sypek ${ }^{2}$ \\ ${ }^{1}$ Institute of Optoelectronics, Military University of Technology, Kaliskiego 2, 00-908 Warszawa, \\ ${ }^{2}$ Faculty of Physics, Warsaw University of Technology, Koszykowa 75, 00-662 Warszawa
}

Received August 12, 2012; accepted September 24, 2012; published September 30, 2012

\begin{abstract}
Detection of about 20 ns pulses by a fast and sensitive NbN-based Hot Electron Bolometer is reported. The pulses were generated by an Optical Parametric Oscillator operating in a non collinear, intersecting cavity configuration. We present details of the oscillator and bolometer as well as the measurement setup. The linearity of the system was measured and agrees well with theoretical considerations.
\end{abstract}

Terahertz radiation $(0.1-10 \mathrm{THz})$ has been the focus of attention of researchers for over three decades due to its interesting properties. Unfortunately, this range of electromagnetic spectrum suffers from a lack of cheap and efficient sources and detectors as well as ready-to-use systems and devices. Complicated and expensive Time Domain Spectroscopy (TDS) is commonly applied in the $\mathrm{THz}$ range because of its broad frequency range $(0.1-3.0 \mathrm{THz})$ and sensitivity [1]. Photomixing $\mathrm{cW}$ systems with tunable diode lasers is cheaper than TDS and more compact, but its primary disadvantage is its relatively low output power compared with other techniques of continuous wave $\mathrm{THz}$ generation and rather narrowband operation in comparison to TDS [2].

Terahertz Optical Parametric Oscillators (OPO), except for extremely big and expensive synchrotrons and free electron lasers, are the only sources which can be tuned in to the comparable range.

In this paper we report the detection of fast and intensive $\mathrm{THz}$ pulses by a very fast and sensitive bolometer. The THz OPO systems usually require short high peak power pulses from a pump laser to reach the region of second order nonlinear optical interaction, which enables generation of $\mathrm{THz}$ pulses. To achieve maximum sensitivity, such pulses, usually nanosecondlong, must be detected by fast detectors with low noise equivalent power (NEP). Therefore, we applied a commercially available OPO and a hot electron bolometer (HEB) to investigate the system performance and capabilities.

The OPO from MSquared company is a parametric oscillator that converts an input laser wave from a Qswitched Nd:YAG laser (1064nm) into the lower frequency radiation from the $\mathrm{THz}$ range thanks to the

\footnotetext{
*E-mail: npalka@wat.edu.pl
}

nonlinear optical interaction in a crystalline gain medium $\left(\mathrm{MgO}: \mathrm{LiNbO}_{3}\right)$ [3].

The frequency of the $\mathrm{THz}$ radiation can be tuned by changing the angle between the pump and OPO cavity axes. This angle is changed electro-mechanically and under computer control. Generating the $\mathrm{THz}$ radiation in cavity geometry also has the advantage that the $\mathrm{THz}$ beam has a near-diffraction-limited spatial mode quality [3]. The more detailed description of the OPO can be found in [4].

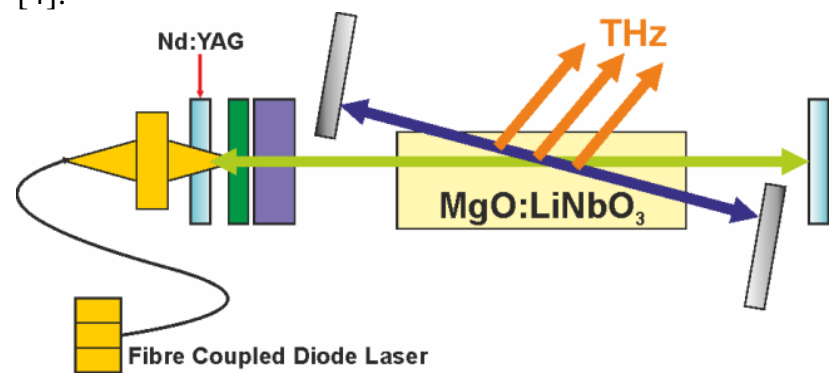

Fig. 1. Schematic illustration of Firefly [3].

The laser can be tuned in to the range of $0.7-2.5 \mathrm{THz}$; its repetition rate equals to $53 \mathrm{~Hz}$, the duration of the pulse is about $10 \mathrm{~ns}$, its energy is $10 \mathrm{~nJ}$ and spectral width $50 \mathrm{GHz}[3]$.

The tuning curve of the OPO (Fig. 2) was measured by means of the Golay cell in the setup described in [5]. Due to poor signal intensity the measurements were limited to the $0.7-2.2 \mathrm{THz}$ range. The drops in signal intensity at 1.1, 1.4 and $1.7 \mathrm{THz}$ are connected with water vapor attenuation.

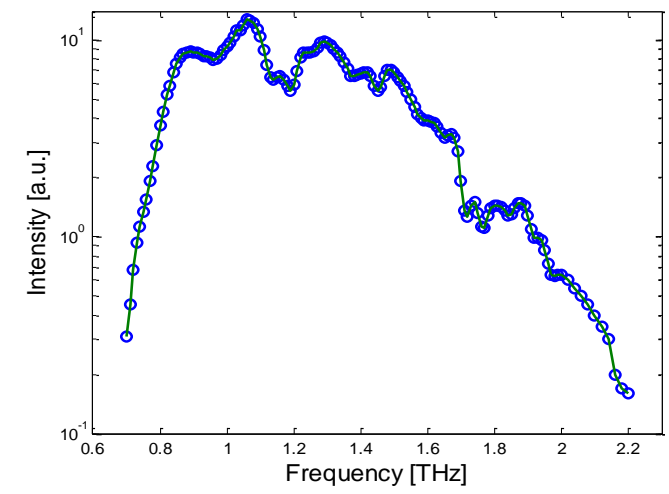

Fig. 2. Tuning curve of the OPO laser [5] 
HEB from Scontel, Russia, operates at a temperature of about $8.8 \mathrm{~K}$ in a cryogenic refrigeration system which is based on a closed-loop helium expansion cycle. The sensitive element is a bridge from a 4-nm thick $\mathrm{NbN}$ (niobium nitride) film integrated with a planar logarithmic spiral antenna on high-resistivity silicon [6]. HEB works in the $0.3-3 \mathrm{THz}$ range with NEP $\sim 3 \times 10^{-13} \mathrm{~W} / \mathrm{Hz}^{1 / 2}$ and the operating range of $0.1 \mu \mathrm{W}$. Thanks to exploitation of hot electrons in a superconducting state, the detector is very fast with a minimum response time equal to 50ps. A cryogenically-cooled HEMT amplifier works in the $1-3500 \mathrm{MHz}$ range with a gain of $25 \mathrm{~dB}$. The $\mathrm{THz}$ radiation is focused with a silicon lens, and then is coupled to a sensitive bolometer using a planar antenna (Fig. 3).

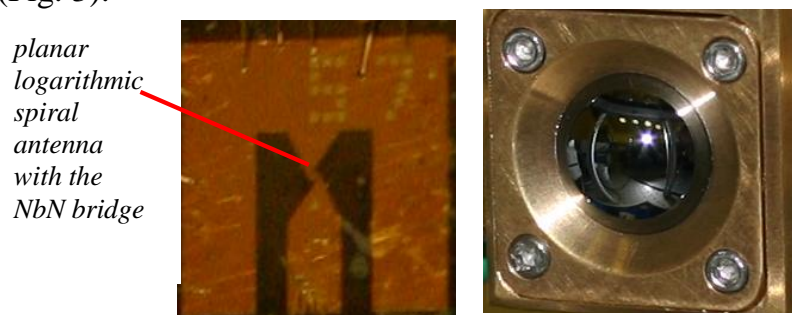

Fig. 3. HEB: the planar logarithmic spiral antenna with an $\mathrm{NbN}$ bridge (left) and a silicon lens (right).

The heart of the detector is the superconducting structure made of $\mathrm{NbN}$, which is a low temperature superconductor with a critical temperature $\left(\mathrm{T}_{\mathrm{C}}\right)$ of about 8K. To avoid problems with cryoliquids we used a Two Stage Pulse Tube Refrigerator (PTR). Its cooling power (2nd Stage and 1st Stage combined 0.25W @ 4.2K with $10 \mathrm{~W} @ 65 \mathrm{~K})$ is sufficient for cooling the structure even when it is heated by radiation coming through the window. The PTR cryocooler was chosen because it has no moving parts in the low temperature part of the device in contrast to other cryocoolers (e.g. Gifford-McMahon). It ensures very low vibrations of the bolometer (less than 5 microns), which is crucial for its performance.

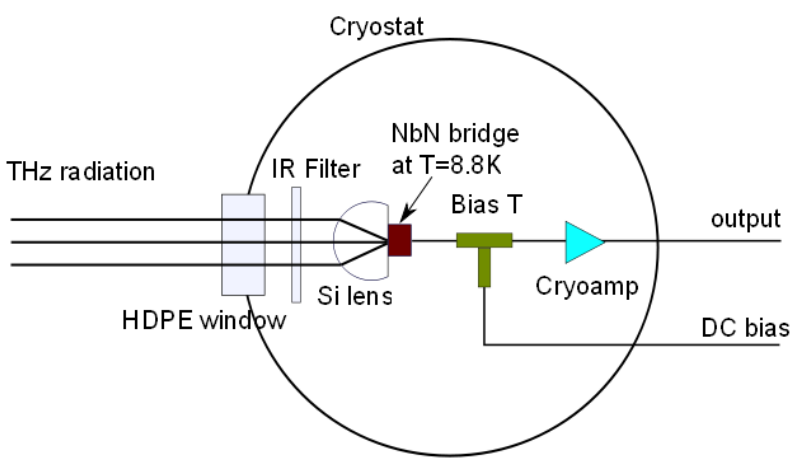

Fig. 4. Optical and electrical scheme of the bolometer.

HEB was applied as a detector of pulses from the OPO. Its sensitive element is the bridge from a 4-nm thick $\mathrm{NbN}$ film integrated with a planar logarithmic spiral antenna on an insulating substrate (high resistivity silicon). The $\mathrm{NbN}$ film was manufactured using a DC magnetron sputtering technique. The required configuration of the device is created by photo- and e-beam lithography followed by chemical and ion etchings. The HEB chip is mounted on the bolometer holder with a silicon lens (Fig. 3). One of the contact pads of the sensitive element is connected by ultrasonic bonding to the central conductor of the $50 \Omega$ microstrip transmission line, the other one is connected to the ground.

The bolometer detector is installed into a holder which is mounted onto a cold plate of the cryorefrigerator Janis PTCM-4-3. Signal radiation is coming into a refrigerator through its input window made of high density polyethylene (HDPE) and IR filter. The IR filter is mounted on the temperature screen of $50 \mathrm{~K}$. To avoid saturation, the power of the incoming radiation must not exceed $0.1 \mu \mathrm{W}$.

During the operation of the detector unit, the sensitive element is heated to a temperature close to the critical temperature of the superconductor (required accuracy is $0.01 \mathrm{~K}$ ), then it is biased with an optimal current.

The response to modulated radiation is the voltage oscillations at the output of the sensitive element. These oscillations are amplified at the output of the detector unit. A biased T-adapter is exploited to decouple DC circuits and a signal channel (Fig. 4).

During the characterization procedure, we choose the region of voltages $(\sim 1.85 \mathrm{mV})$ shown in Fig. 5. For these voltages the output signal is the highest and the most stable. The marked region is also optimal, taking into account noises as it was suggested in [7]. For the chosen voltage the detector also has the best sensitivity.

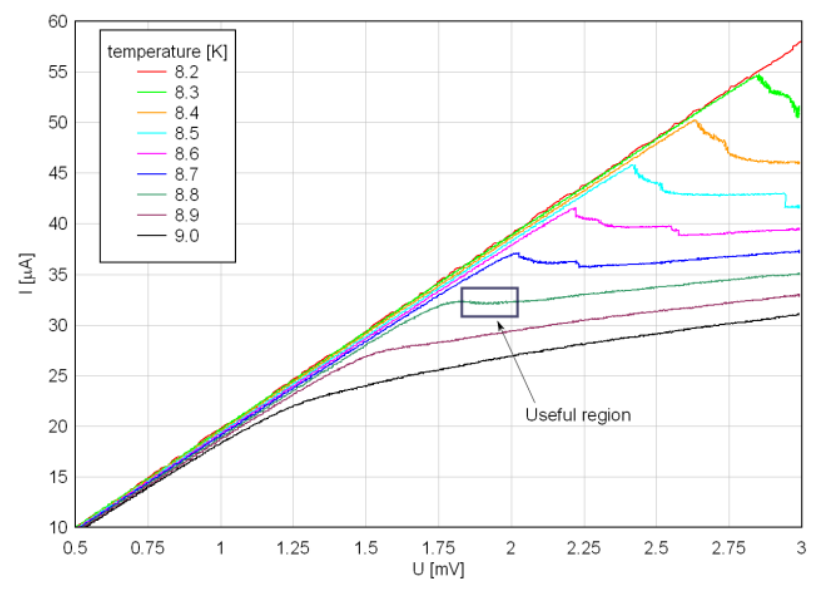

Fig. 5. Current-voltage characteristics measured for various temperatures near superconducting transition.

To measure fast pulses from the OPO we built an arrangement, which is presented in Fig. 6. The $\mathrm{THz}$ 
radiation from the OPO, through gold mirrors (M), polarizers (P1, P2) and attenuator (A) is coupled to the detector. Since HEB is very sensitive and could be easily saturated, we strongly attenuated the radiation up to $10^{-4}$ by polarizer P1 and attenuator A. Polarizer P2 can be used to measure the linearity of the system. The distance between the source and the detector was about $3.5 \mathrm{~m}$.

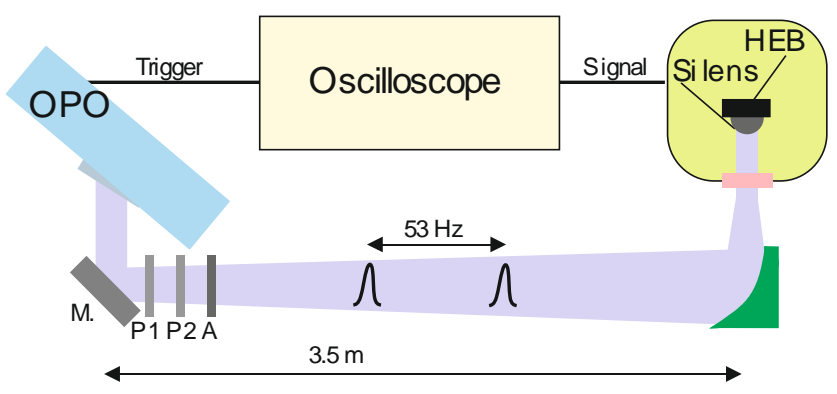

Fig. 6. Measurement setup.

The Full Width at Half Maximum of the recorded pulses measured with a fast digital oscilloscope (10GS/s) is about 20ns (Fig. 7), which agrees well with the manufacturer's data. The pulses are distorted by high voltage disturbances connected with Q-switching of the OPO pump laser.

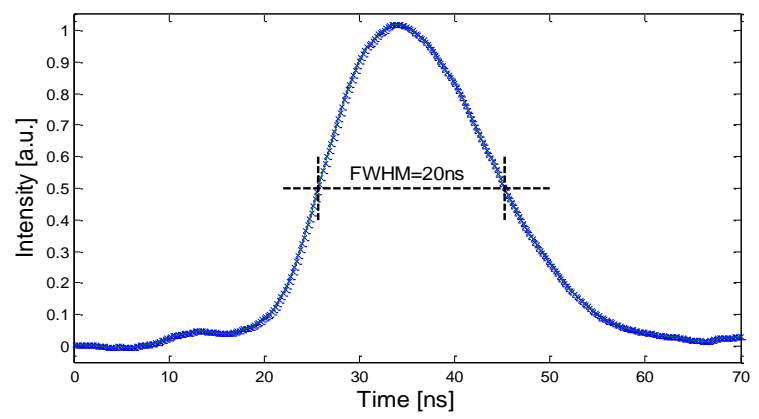

Fig. 7. Pulse measured by HEB

Moreover, we measured the linearity of the detector rotating the polarizer axis in the range of $-90:+90$ degrees. The pulses were averaged and integrated for better stability. We obtained a good similarity to the theoretical curve of the polarizer (Fig. 8), where the intensity of radiation is proportional to $\cos ^{2} \phi$, where $\phi$ is the angle between the $\mathrm{THz}$ radiation polarization direction and the axis of the polarizer.

For the incoming radiation weaker than $0.8 \mathrm{nW}$ the signal-to-noise ratio became lower than one. It means that the dynamics of our system was only a little better than $20 \mathrm{~dB}$. To improve this parameter of our system we used a set of attenuators with precisely measured attenuation. It allows us to enhance dynamics to almost 50dB.

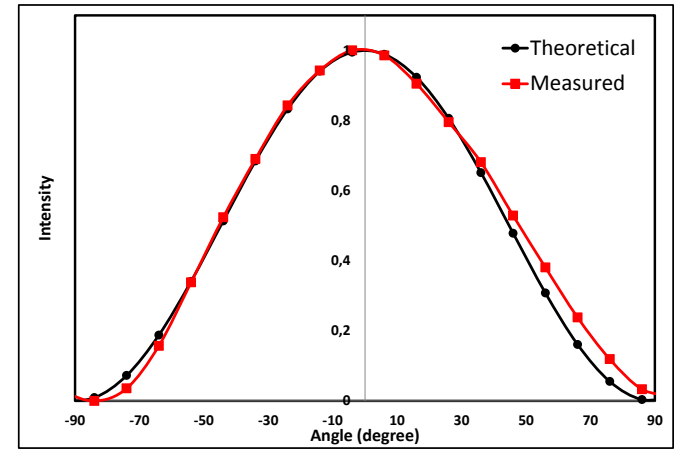

Fig. 8. Linearity of the detector measured by the polarizer.

The system with a tunable source and a broadband and sensitive detector is planned to be applied for stand-off spectroscopy (reflection configuration) of dangerous materials, including explosives. It is commonly known that some explosive materials, like Hexogen, Penthryte and Octogen have distinctive spectroscopic features (called "fingerprints") roughly in the range of $0.8-2.5 \mathrm{THz}$ [5]. Therefore, stable tunability in this range with a step of about $5-10 \mathrm{GHz}$ is desired for this application. According to our calculations, a detector with a very high dynamic range $(60-70 \mathrm{~dB})$ and minimum response time equal to $1 \mathrm{~ns}$ is required to measure fast pulses from the OPO. The interaction of $\mathrm{THz}$ waves with a tested sample is complicated due to many factors like: attenuation of the atmosphere, surface of the sample (specular or diffuse reflection) and distance to the sample.

The proposed system is fast and provides possibility to measure $\mathrm{THz}$ nanosecond pulses in real time in contrast to TDS systems, where pulses are "synthesized" in a complicated way using a scanning delay line [1]. This feature renders the OPO-HEB configuration ideal for realtime security applications. A scanning mirror could direct the radiation reflected from the object to the HEB detector, which provides fast spectroscopic information about the threats.

The Project was financed by the Polish Ministry of Science and Education under the Project O N515 020140.

\section{References}

[1] L. Yun-Shik, Principles of Terahertz Science and Technology (Springer, 2008).

[2] E.F. Plinski, Bull. Pol. Ac.: Tech. 58, 463 (2010).

[3] Firefly-THz User Manual (M Squared Lasers Ltd, 2011).

[4] A. Sato et al., Rev. Sci. Instrum. 72, 3501 (2001).

[5] N. Palka et al., Proc. of MIKON 2012, 265 (2012).

[6] Scontel, Superconducting Nanotechnology, HEB User Manual.

[7] A.D. Semenov et al., J.App. Phys. 88, 1 (2000). 